\title{
Früherkennung lohnt sich auch über 75
}

\section{Bislang galt, dass Prostatakrebs bei älteren Männern langsamer wächst und dass die Patienten meist aus anderen Gründen sterben. Doch in einer Studie zeigte sich jetzt: Über die Hälfte aller Prostatakarzinom-Todesfälle betrifft über 75-Jährige, obwohl hier nur ein Viertel aller Tumoren diagnostiziert wurde.}

n der Studie wurde untersucht, wie häufig in verschiedenen Altersgruppen zur Diagnose bereits Metastasen vorlagen und ob ein Zusammenhang zwischen Alter und prostatakrebsspezifischer Mortalität bestand. Hierzu wurden die Daten von über 460.000 Amerikanern ausgewertet, bei denen 1998-2007 ein Prostatakarzinom festgestellt worden war.

Die Zahl neu diagnostizierter Tumoren stieg mit dem Alter an, erreichte bei den 70-Jährigen ein Maximum und fiel danach wieder ab. Dies galt allerdings nicht für fortgeschrittene Fälle mit Metastasen (M1). Je älter die Patienten bei Diagnose waren, desto häufiger lag ein GleasonScore zwischen 8 und 10 sowie ein M1Stadium vor: Während sich dies bei den unter 75-Jährigen bei Diagnose nur bei $3 \%$ fand, waren es bei den 75- bis 79-Jährigen bereits $5 \%$, zwischen 80 und 84 Jahren $8 \%$, bei den 85 - bis 89 -Jährigen $13 \%$ und ab 90 Jahren $17 \%$. Entsprechend stieg mit dem Alter die kumulative Inzidenz der Todesfälle infolge des Prostatakarzinoms an. Während sie bei den Patienten, die jünger als 75 Jahre waren, bei 3-4\% lag, starben innerhalb von fünf Jahren $7 \%$ der Patienten zwischen 75 und 79 an ihrem Krebs $(21 \%$ an anderen Ursachen, aU), $13 \%$ aus der Gruppe der 80 - bis 84-Jährigen ( $32 \% \mathrm{aU}), 20 \%$ der 85 - bis 89-Jährigen ( $43 \% \mathrm{aU})$ und $30 \%$ der Patienten $\geq 90$ Jahre $(55 \% \mathrm{aU})$.

Obwohl nur 26\% der Patienten, bei denen ein Prostatakarzinom diagnostiziert wurde, 75 Jahre oder älter waren, stellten sie fast die Hälfte der Gruppe (48\%), in der ein M1-Stadium diagnostiziert wurde. Und auch in der Gruppe der Krebspatienten, die an ihrem Prostatakarzinom starben, waren die Senioren ab 75 mit einem Anteil von $53 \%$ überrepräsentiert.

Fazit: Möglicherweise wachse bei älteren Männern der Prostatakrebs aggressiver als bisher angenommen, so die Autoren. Allerdings könnte auch eine Rolle gespielt haben, dass Männer über 75 seltener an Früherkennungsuntersuchungen teilnehmen und bei erhöhten PSA-Werten nicht so häufig biopsiert würden wie Jüngere. Vor allem vor dem Hintergrund der Diskussion zum Prostatakarzinom-Screening sind diese Ergebnisse interessant, denn sie könnten auf den präventiven Nutzen einer frühen Diagnostik auch bei älteren Männern hinweisen. Christine Starostzik

Scosyrev E et al. Prostate Cancer in the Elderly. Cancer. 2011; doi: 10.1002/cncr.26392.

\section{mRCC: Metastasenfrei dank gezielter Therapie}

Tyrosinkinase-Inhibitoren (TKI) verlängern bei Patienten mit metastasiertem Nierenzellkarzinom (mRCC) das progressionsfreie Überleben. Und wie sieht es mit Komplettremissionen (CR) aus?

Eranzösische Wissenschaftler werteten - in einer retrospektiven Multicenterstudie die Daten von mRCC-Patienten aus,

die zwischen November 2005 und Dezember 2009 in Frankreich und der Schweiz TKI allein oder in Kombination mit loka-

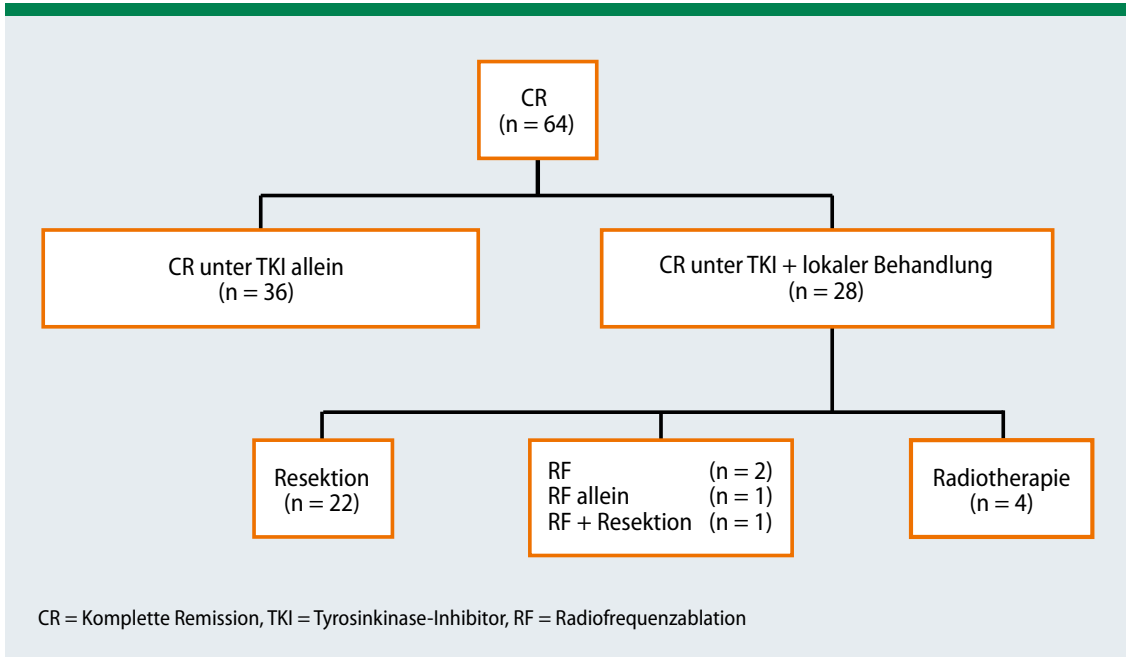

Abb.: 64 Patienten, die eine komplette Remission unter Sunitinib oder Sorafenib erreichten, wurden in 15 Zentren von November 2005 bis Dezember 2009 identifiziert. len Therapien erhalten hatten (Abb.). Bei 64 Patienten $(1,7 \%)$ wurde eine CR erzielt. 60 Patienten waren an einem klarzelligen Tumor erkrankt, vier an einem papillären. Allen war die Niere entfernt worden. Der Großteil der CR-Patienten $(n=59)$ hatte Sunitinib eingenommen. Von den $36 \mathrm{~Pa}$ tienten, die allein systemisch behandelt wurden, beendeten 28 die Therapie bei CR. Im Durchschnitt dauerte es 12,6 Monate bis die Metastasen radiologisch nicht mehr nachweisbar waren. 17 Patienten, die die Einnahme beendeten, sind auch nach einem medianen Follow-up von 255 Tagen in CR. Bei den 28 Patienten, die kombiniert therapiert wurden, vergingen im Durchschnitt 18,5 Monate bis zur CR. 25 brachen danach die Therapie ab. Davon sind 12 auch nach median 322 Tagen tumorfrei.

Fazit: Für Empfehlungen zum Absetzen oder Weiterführen von TKI ist es zu früh. Erst muss man nach Kriterien suchen, anhand derer man das individuelle Rückfallrisiko abschätzen kann. Dagmar Kraus

Albiges L et al. Complete remission with tyrosine kinase inhibitors in renal cell carcinoma. J Clin Oncol. 2012;30(5):482-7. 